\title{
Enhancing the utilization of packed red blood cells stock in maternity hospitals
}

Waleed M. Bawazir, MSc, PhD, Fahad M. Dakkam, BSc, MSc.

\begin{abstract}
الأهداف : تقييم وتحسين كفاءة خدمات نقل الدم في مستشفيات الولادة.

المنهجية : تمت هذه الدراسة على مرحلتين. المرحلة الأولى، واستمرت ثلاثية

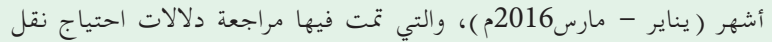

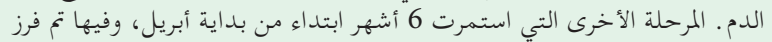

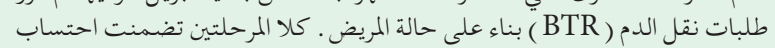

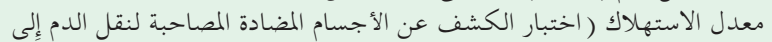

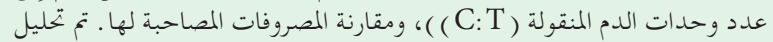

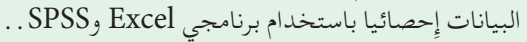

النتائج: حللنا BTRs 1،200، تضم 659 قبل تنفيل تنفيذ السياسة التصحيحية و 541 طلب نقل الدم بعد تنفيذ السياسة التصحيحية .

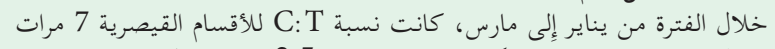

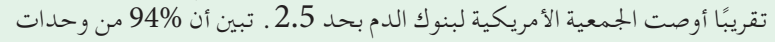

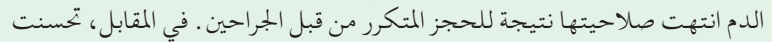

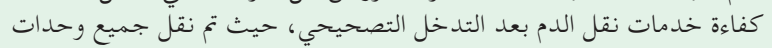

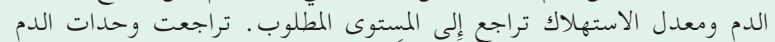

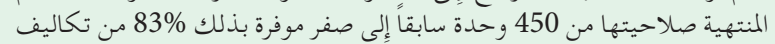

خدمات بنك الدم مقارنة بما قبل التدخل التحان التصحيحي.

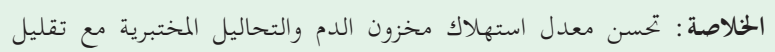

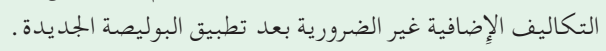

Objectives: To assess and enhance the efficiency of transfusion services in maternity hospitals.

Methods: A case control study was conducted from January to December 2016. A corrective policy of replacing preoperative type and hold step with blood transfusion request (BTR) hold was used only on healthy patients undergoing elective cesarean sections (c-section). The crossmatch/transfusion (C:T) ratio and a cost comparison were the evaluating factors. Data were analyzed using an Excel spreadsheet and SPSS statistical software.

Results: A total of 1,200 BTRs were analyzed, comprising 659 before implementation of the corrective policy and 541 blood transfusion requests after implementation of the corrective policy. From January to March, the C:T ratio of c-sections was nearly 7 times the American Association of Blood Banks recommended limit of 2.5. Most of the blood units (94\%) were damaged due to repeated booking. After implementation, the cost-effectiveness of erythrocyte transfusion was greatly enhanced as all the ordered blood units were used and the C:T ratio was reduced to the ideal limit of one. The number of destroyed units was drastically decreased from 450 units to zero; as a result, $83 \%$ of the transfusion costs were saved.

Conclusion: The policy enhances the cost-effectiveness of erythrocyte transfusion and laboratory testing, and saves on additional, unnecessary costs.

Keywords: erythrocyte transfusion, cesarean section, cost effectiveness

Saudi Med J 2020; Vol. 41 (6): 628-634 doi: 10.15537/smj.2020.6.25084

From the Medical Laboratory Technology Department, Faculty of Applied Medical Sciences, King Abdulaziz University, Jeddah, Kingdom of Saudi Arabia.

Received 3rd September 2019. Accepted 19th April 2020.

Address correspondence and reprint request to: Dr. Waleed M. Bawazir, Medical Laboratory Technology Department, Faculty of Applied Medical Sciences, King Abdulaziz University, Jeddah, Kingdom of Saudi Arabia.E-mail: wbawazir@kau.edu.sa

ORCID ID: https://orcid.org/0000-0002-9393-1538

Disclosure. Authors have no conflict of interests, and the work was not supported or funded by any drug company. 
B lood transfusion services are one of the most important treatment services in daily clinical practice, but are not without cost. Laboratory and blood transfusion testing consumes more than $\$ 65$ billion per annum in the United States. ${ }^{1}$ One cause of this is excessive preoperative ordering of blood components; such as blood units for patients undergoing surgical operations. ${ }^{2}$ This can lead to shortages in blood bank inventories, waste of reagents, and therefore financial burdens on transfusion services. ${ }^{3-5}$ Additionally, a stock of reserved crossmatched units are held on standby for a period of time, after which they may expire and be discarded, further increasing the waste. ${ }^{6}$ This was recently observed in obstetric hemorrhage cases where nearly half of the erythrocyte units ordered for transfusion were not used and were returned to the blood bank. ${ }^{7}$ For this reason, transfusion service providers have implemented strategies to improve transfusion services. The maximum surgical blood order schedule has been proposed to limit the crossmatched to transfused units ratio (C:T) to lower than $2.5 .^{8}$ Other strategies were also used, such as increasing transfusion policy awareness among the medical staff, and implementing the lean 6 sigma methodology. ${ }^{6,9}$ One of the more effective strategies of reducing unnecessary use involved limiting the blood transfusion request (BTR) received by the blood bank to typing and screening of a blood unit, rather than typing and crossmatching. Alghamdi et al ${ }^{10}$ recommended the use of type and screen approach on preoperative requests for cardiac operations to improve the $\mathrm{C}: \mathrm{T}$ ratio by $66 \%$. This also suggests that there is some latitude for improving the preoperative blood ordering and blood testing by reviewing the indications for transfusion and limiting unnecessary testing.

It has been reported that elective cesarean sections (c-section) are less risky than vaginal deliveries; however, the orders and crossmatching for blood transfusion for elective procedures can be excessive. .,11,12 $^{\text {, }}$ A study revealed that only a small number of the blood units reserved for elective c-sections are used $(0.5 \%$, $\mathrm{n}=7809) .{ }^{5}$ This suggests that blood banks have to provide excessive units for elective c-sections and can be exposed to shortages. Some hospital facilities, such as surgical teams, and anesthesiologists seek to improve their practical performance without considering the burden on blood bank resources caused by unnecessary BTRs. ${ }^{9}$ In Saudi Arabia, blood transfusion services are hospital-based blood banking systems that are financially supported by the government. Frequent blood grouping and reservation of blood units, which are never used, results in a waste of blood bank resources. Previous studies of blood transfusions for c-sections either considered or recommended policies to limit the utilization of blood units to the type and hold step to reduce waste. ${ }^{5,13}$ However, they did not address the effects of holding the BTR for healthy patients on the increased consumption of costly reagents in blood typing and compatibility testing.

The aim of this study was to reduce the judicious usage of transfusion services by evaluating a new corrective policy designed to replace the "type and save" step. For healthy patients undergoing elective c-sections, this policy is to consist of only holding the BTR (no testing). Findings will be compared before and after the implementation of this corrective policy.

Methods. The study was conducted at Abha Maternity and Children's Hospital (AMCH), Abha, Saudi Arabia. A paper-based, referral, and educational hospital with a capacity of 240 beds; and, is in the Southern region of Saudi Arabia. Informed consent to access blood bank records and data from the maternity hospital patients was conducted by the referring hospital. We obtained approval from the referring hospital to use these data and ethical approval from the Research Review Committee at the Faculty of Applied Medical Sciences (no. 64886/37). Patient data were anonymized before analysis, according to the Saudi Ministry of Health regulations and the principles of the Declaration of Helsinki.

Abha Maternity and Children's Hospital, Abha, Saudi Arabia was selected due to inefficient blood unit utilization caused by the unnecessary reservation of blood units for c-sections. These blood units are commonly not used and are sometimes discarded due to passing the expiration dates. This study aimed to optimize the usage of blood units in order to improve blood bank inventory and efficiency by reducing unnecessary ordering for patients who may not need erythrocyte transfusions. ${ }^{5}$ The design of the study was based on a review of all the documents related to the work policies of the blood bank: both the internal policies, and the policies which regulate relationships with other departments. Data collection depended on the classification of blood transfusion requests according to the patient's health status as well as the number of required units. This study took almost 3 months due to the absence of a computer system in the hospital. The sources of information were paper records and archived blood transfusion forms.

A retrospective cohort study from January 2016 to March 2016 was used to analyze routinely collected maternity BTRs at the blood bank laboratory archive. Preoperative maternity BTRs $(n=1198)$, including 
659 BTRs for elective c-sections, were reviewed and classified; and, hemoglobin concentrations or placental issues, and the total number of crossmatched and transfused blood units were recorded.

The findings of all collected data were summarized and explored. The retrospective C:T ratio for elective c-sections was used as a control and compared to the $\mathrm{C}: \mathrm{T}$ ratio after the implementation of the "save and type" policy.

Prospective analysis after the newly implemented corrective policy on c-section BTRs. The prospective analysis (from April 2016 to December 2016) was a more comprehensive assessment of BTRs $(n=541)$ as per the instructions of the anesthesia department, which dictated blood unit reservations as a condition for patient inclusion in the schedule of operations. Prior to policy implementation, $\mathrm{AMCH}$ policy required that all preoperative BTRs must be typed, screened, crossmatched, and reserved for a maximum of 3 days prior to c-sections. The new policy classified the maternity BTRs into 2 types based on the hemoglobin concentration and the expected risk during or after operation. Preoperative BTRs for patients with a hemoglobin of $\leq 12 \mathrm{~g} / \mathrm{dl}$ or a diagnosis of risk factors such as placenta previa or accreta, a previous abortion, acute hemorrhage, or multiple pregnancies were excluded from the corrective policy and considered at risk. Vaginal births and emergency cases were also excluded from the study. Blood transfusion requests included in the corrective policy were those for patients undergoing elective c-sections, with a hemoglobin concentration of $>12 \mathrm{~g} / \mathrm{dl}$ and no or low risk factors such as umbilical cord prolapse, or small maternal pelvis. The requested units for healthy and uncomplicated cases (depending on retrospective findings, those who rarely received blood) were excluded from the "type and save" protocol. Alternatively, BTRs were received and put on hold without typing of the erythrocyte unit(s), known as a "receive and hold". If a blood transfusion was required intra- or post-operatively, the BTRs was treated as an emergency and the blood unit(s) crossmatched by immediate spin depending on the 28th-week of pregnancy results of allo-antibody screening. This type of BTR was excluded. Bleeding BTRs were treated according to the emergency hemorrhage protocol, and subsequently massively transfused with uncrossmatched units. This new policy was implemented to improve the cost-effectiveness of the blood bank by reducing the costly consumption of reagents and erythrocyte unit waste caused by preoperative non-risky c-section BTRs. Auditing of reagent consumption, transfusion costs, and the number of crossmatched, transfused units was performed continuously to assess improvements in efficiency.

Patients with a hemoglobin of $\leq 12 \mathrm{~g} / \mathrm{dl}$ or a diagnosis of risk factors such as placenta previa or accreta, a previous abortion, acute hemorrhage, or multiple pregnancies were excluded from the corrective policy and considered at risk. Vaginal births emergency cases were also excluded from the policy. Patients undergoing elective c-sections, with a hemoglobin concentration of $>12 \mathrm{~g} / \mathrm{dl}$ or have no or low risk factors such as small maternal pelvis and umbilical cord prolapse were included in this policy.

Statistical analysis. Data were analyzed via Excel $^{\oplus}$ 2010 (Microsoft $^{\oplus}$, Redmond, Washington, U.S.A.) spreadsheets or the Statistics Package for Social Sciences for Windows, version 22 (IBM Corp, Armonk, NY, USA). The C:T ratio was used as transfusion services efficiency indicator. A 2-tailed Chi-squared test (1 degree of freedom [df.]) was used to examine whether there was no difference between the total number of maternity and c-section operations patients before and after the implementation of the corrective policy. The test was also used to examine whether there was no difference between the total number of risky and non-risky patients before and after implementation. A one-tailed McNemars' Chi-squared test (df. 1) for paired data with Yates correction was used to examine whether the BTR holding policy significantly reduced the number of crossmatched units in comparison to the period before implementation. A similar test was also used to examine whether the BTR holding policy significantly reduced the number of expired units. A $p$-value of $<0.05$ was statistically significant.

Cost estimation. The financial value of the total number of ID gel cards and reagents (DiaCell I-II-III, $\mathrm{ABO} / \mathrm{RhD}+$ Reverse Grouping, Liss/Coombs) used for each BTR was calculated according to the price quotation submitted by the manufacturers. All reagents used for $\mathrm{ABO}$ typing, and antibody screening and identification were obtained from BioRad (BioRad, Hercules, California, USA).

Calculation of blood unit cost. [Total number of (crossmatched, or transfused or expired) blood units $\mathrm{x}$ cost of a single unit $(\$ 133)]$

Calculation of crossmatch and group and hold cost. The total cost of type and hold calculation: [Total number of patients + total transfused + un-transfused + wasted blood units) X (cost of ID gel cards + reagents of DiaCell I-II-III, ABO/Rh+ reverse grouping (\$2.65)]

Crossmatch calculation: [Total number of patients + total transfused + un-transfused + wasted blood units) X 
(cost of ID gel cards + reagents of Liss/Coombs $(\$ 0.98$ $+\$ 0.37))]$

Results. Patients demographics are shown in Table 1. The blood bank laboratory received BTRs for 1,200 patients undergoing c-section operations from January to December 2016. The patients' ages ranged from 21 to 37 years with a mean hemoglobin concentration of $12 \mathrm{~g} / \mathrm{dl}$ (normal range $=12$ to $15.5 \mathrm{~g} / \mathrm{dl}$ ). Overall, there was no significant difference in the total number of maternity and c-section patients before and after the implementation of the study $\left(\chi^{2}(1)=3.65, p=0.056\right)$. Of these patients, approximately $55 \%$ underwent c-sections from January to March 2016. Before the implementation policy, all of the c-section patients reserved erythrocyte transfusions. After the implementation of the BTR sorting policy, $81.9 \%$ of c-sections were healthy and their BTRs were held without further testing.

Figure 1 shows the number of BTRs, crossmatched, and transfused units during the 3 months before the implementation of the corrective policy. Of all BTRs received by the blood bank, 55\% were for patients admitted for a c-section. The BTRs for c-section patients reserved the majority (76.4\%) of crossmatched units for all maternity operations, but only $5.4 \%$ were actually transfused. Sorting of BTRs according to patients' hemoglobin concentration excluded the BTRs of healthy women undergoing elective c-section from routine compatibility tests. Consequently, a significant reduction in blood utilization was observed, and 100\% of crossmatched blood units were transfused unlike before implementation (McNemars' $\chi^{2}(1)=55.408$, $p<0.001$ ) (Figure 2). Hence, the $\mathrm{C}: \mathrm{T}$ ratio was significantly reduced to 18 times that of before the implementation, reducing the overall $\mathrm{C}: \mathrm{T}$ ratio for all maternity operations to nearly the ideal value of one.
Table 2 displays the impact of the new policy on the estimated total costs of the crossmatched, transfused, and expired units as well as the total cost of routine testing pre- and post-correction. In view of the precorrection cost of the crossmatched and transfused units, a difference of $\$ 179,683$ between the 2 totals was observed. The total cost of 450 units that expired due to re-reservation exceeded the cost of the precorrection transfused units by more than 5 times. After implementation of the corrective policy, the number of expired units was significantly reduced to 0 in

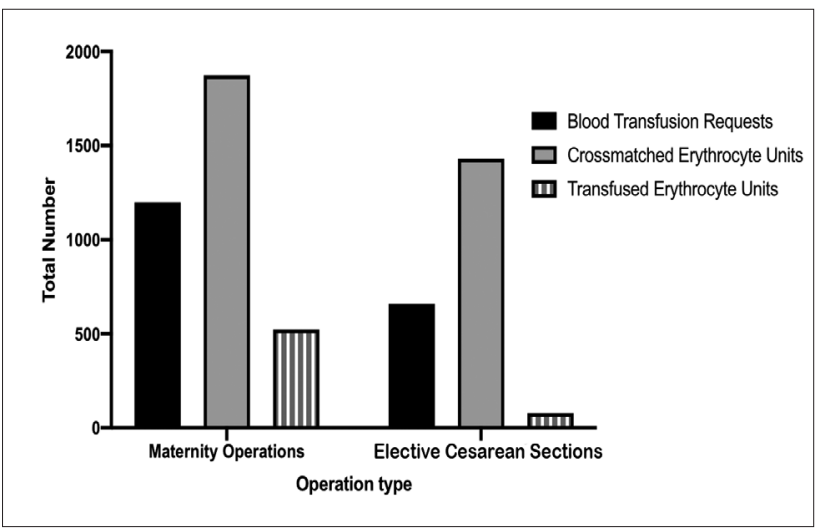

Figure 1 - The utilization of erythrocyte units for maternity operations at Abha Maternity and Children's Hospital (AMCH), Abha, Saudi Arabia in the pre-implementation period. Includes cesarean section (c-section) operations before the implementation of blood transfusion request (BTR) holding policy, and the omission of the type and hold step at $\mathrm{AMCH}$ (January to March 2016). A total of 1,198 BTRs were received in the AMCH blood bank which reserved 1871 crossmatched units. Of all the maternity operations BTRs, 659 BTRs were for c-section operations which reserved 1429 crossmatched units of all maternity operations at AMCH. However, only 78 of these erythrocyte units were actually transfused to elective c-section patients.

Table 1 - Demographics of patients in the study from January to December 2016.

\begin{tabular}{|c|c|c|c|c|}
\hline Patients' operation & $\begin{array}{l}\text { Total number of } \\
\text { patients from Jan } \\
\text { to Mar } 2016\end{array}$ & $\begin{array}{c}\text { Total number of } \\
\text { patients from April to } \\
\text { December } 2016\end{array}$ & $\begin{array}{c}\text { Two tailed } \\
\text { Chi-squared } \\
P \text {-value }\end{array}$ & $\begin{array}{l}\text { Total number of } \\
\text { patients from January } \\
\text { to December } 2016\end{array}$ \\
\hline Maternity operations & 1198 & 855 & \multirow{2}{*}{0.056} & 2053 \\
\hline Cesarean section operations & 659 & 541 & & 1200 \\
\hline $\begin{array}{l}\text { Cesarean sections for patients having } \\
\text { hemoglobin concentration } \leq 12 \mathrm{~g} / \mathrm{dl} \text { or } \\
\text { high risk factors }\end{array}$ & 263 & 98 & \multirow[b]{2}{*}{$<0.001^{*}$} & 361 \\
\hline $\begin{array}{l}\text { Cesarean sections for patients having } \\
\text { hemoglobin concentration }>12 \mathrm{~g} / \mathrm{dl} \\
\text { with no or low risk factors }\end{array}$ & 396 & 443 & & 837 \\
\hline
\end{tabular}


Table 2 - Total cost of erythrocyte transfusions before and after the implementation of the corrective blood transfusion request holding policy.

\begin{tabular}{|c|c|c|}
\hline Erythrocyte units' costs & $\begin{array}{l}\text { Total costs } \\
\text { in the pre- } \\
\text { implementation } \\
\text { period }(\$)\end{array}$ & $\begin{array}{l}\text { Total costs in the post- } \\
\text { implementation period } \\
(\$)\end{array}$ \\
\hline Crossmatched units & 190,057 & 27,132 \\
\hline Transfused units & 10,374 & 27,132 \\
\hline Expired units due to repeated reservation & 59,850 & 0 \\
\hline \multicolumn{3}{|l|}{ Testing costs } \\
\hline Routine $\mathrm{ABO} / \mathrm{RhD}$ typing and antibody screening & 9,111 & 541 \\
\hline $\begin{array}{l}\text { Routine } \mathrm{ABO} / \mathrm{RhD} \text { typing and antibody screening for expired } \\
\text { units }\end{array}$ & 3,578 & 0 \\
\hline
\end{tabular}

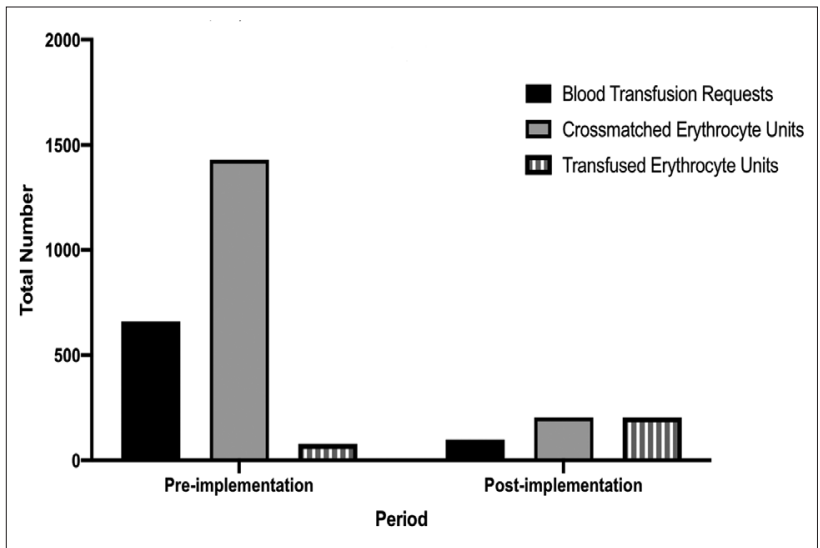

Figure 2 - The cost-effectiveness of erythrocyte transfusion units for cesarean section (c-section) operations pre- and postimplementation of the corrective policy at Abha Maternity and Children's Hospital, Abha, Saudi Arabia (January - December 2016). The corrective policy excluded BTRs of healthy women from routine compatibility tests. The crossmatch/transfusion ratio for c-section operations was 18 fold the recommended C:T limit indicating that overall $94.5 \%$ of the crossmatched units were not transfused. The corrective sorting of BTRs according to the expected need for transfusions significantly increased the efficiency of blood utilization to $100 \%$. Hence, all crossmatched blood units were transfused (McNemars' $\left.\chi^{2}(1)=55.408, p<0.001\right)$.

comparison to the pre-correction period (McNemars' $\left.\chi^{2}(1)=91.781, p<0.001\right)$. Consequently, the total financial cost of all units and tests were decreased after the implementation of the corrective policy. The financial value of testing of the reserved units which were not transfused together with the value of expired units constituted $79 \%$ of the total cost of reagent and materials necessary for routine compatibility testing (antibody screening, ABO typing, and crossmatching).

Discussion. Blood transfusion plays a major role in the management of both hematological disorders and surgical procedures. This widespread need has created a growing demand for blood units; however, not all blood units are pre-emptively ordered for surgery are utilized. ${ }^{2}$ The costs of typing, antibody identification and crossmatching also contribute to the direct expenses of blood banks, especially when the blood is not transfused. ${ }^{5}$ Since 1967, different policies and low cost measures have been used to improve the cost-effectiveness of blood transfusions. ${ }^{6,14,15}$ These measures limited BTRs to one of 3 steps: 1 ) type and crossmatch; 2) type, screen and hold; 3) or type and save. ${ }^{8,10}$ Some countries such as the United Kingdom use the electronic crossmatch as an approach to improve patients' safety as well as the cost-effectiveness of erythrocyte transfusions. ${ }^{16}$ However, this system requires regular blood donors with known history. In other countries, such as Saudi Arabia, the donation pool is mainly compromised of replacement donors which adds more stress to blood banks. The need to prepare blood units for c-section has been a subject for debate. Typing and holding BTRs for healthy elective c-section patients lead to reagents waste and erythrocyte units. Approximately half of the BTRs reviewed in this study were for maternity cases; however, very few of the crossmatched units were utilized. This is because most of the women who undergo elective surgery were in good health and tolerated the amount of blood lost (which does not typically exceed one litre according to some studies) especially with an average hematocrit level not less than $20 \% .^{3-5}$ In addition, BTRs for elective c-sections of healthy, non-high-risk patients do not require routine testing. ${ }^{3}$ This is in concordance with a previous study that found most patients who undergo an elective c-section do not require a blood transfusion., 5 Therefore, the requesting of blood units should be rationalized, so that blood is saved for emergencies. Some studies have examined the practice of type and hold, and the controlled reservation of blood units and have concluded that the practice leads to an increase in the number of typing, screening, and crossmatching tests. ${ }^{18,19}$ Accordingly, the unnecessary 
pre-correction costs of testing increased as shown in Table 2, because the majority of the elective c-section patients did not require blood transfusion. This study points out that the type and hold technique might not be an effective strategy to limit the reservation of units from routine testing for elective c-sections. Therefore, a new policy may be used to improve the cost-effectiveness of erythrocyte transfusion. In this study, the policy of preoperative type and hold was replaced by BTR hold without further testing only for patients undergoing elective c-sections without anemia, placental complications, or emergency transfusions.

A recent study advocated the use of "Deming Plan, Do, Check, and Act cycle" in the management of blood units. This strategy had a positive effect of decreasing the blood unit waste that resulted from the reservation of units, however, it also had the negative impact of increasing the consumption of reagents during the type and screen step. ${ }^{20}$ Reagent waste places a great burden on hospitals, especially those in countries that import reagents. ${ }^{21,22}$ Our approach sorts the BTRs of c-section patients according to health status and improves the efficiency of blood services. The type and hold step was not implemented for elective c-section cases but was kept only for complicated cases, an approach highly recommended in previous studies. ${ }^{5}$ The ideal C:T ratio should be 1.0 , but a ratio below 2.5 indicates an acceptably efficient utilization. ${ }^{22}$ In this study, replacing the type and hold protocol with holding the BTRs for healthy elective c-sections has dramatically improved the $\mathrm{C}: \mathrm{T}$ ratio to one. Other policies reported in previous studies reduced the $\mathrm{C}$ : $\mathrm{T}$ ratio to between 1.5 and 2.5.7,10 The implementation of cost-effective policies may be affected by hospital size, type of operations conducted within the hospitals, and number of admitted patients. ${ }^{23}$ In this study, $50 \%$ to $60 \%$ of healthy pregnant women may choose elective c-sections instead of vaginal delivery. Reaching the C:T ratio of one was possible due to the implementation of the corrective policy on the BTRs for healthy women. Other hospitals may adopt this policy after reviewing the indications for transfusions and the patients' demographics. It might be argued that the healthy c-section patients may require a blood transfusion intraoperatively or post-operatively and that this policy may compromise patients' safety. Patients that meet these criteria would follow the emergency protocol of the hospital and would not be affected by the BTR holding policy. Additionally, no adverse outcomes due to the unavailability of blood units were recorded in this study, and most of the patients left the hospital within 24 hours of the operation. The financial cost assessment indicates the success of these measures.
The reduction from the previous costs were significant. Importantly, the number of non-transfused units reserved for c-sections were significantly reduced. This reduction may have a positive effect on all blood groups in general including O-negative erythrocyte units. Some blood transfusion organizations recommend that O-negative units used should not exceed $10 \%{ }^{20}$ Eliminating reservation increased the possibility of units being transfused, and reduced wasting of units which may include the rare $\mathrm{O}$-negative units. It is worthwhile to emphasise the large number of units wasted pre-implementation of the study compared to the period after the policy implementation.

Study limitations. The preoperative erythrocyte transfusion requests for healthy patients undergoing elective c-sections in a maternity hospital did not consider intraoperative or post-operative erythrocyte transfusion. During the study, we noticed a gap in the interface between the surgeons, and the blood bank, which is theorized to also be a reason for the over-ordering of blood units. For this limitation, ways to bridge this gap are needed to be investigated with new ethical approval. Studying the implementation of this policy on other maternity hospitals may highlights other variables contributing to erythrocyte waste; however, this was not possible to conduct due to time and logistical restrictions. The impact of this policy on individual erythrocyte blood groups and other blood components should be thoroughly studied. Future comparative studies on the erythrocyte transfusion policies in maternity and other specialist hospitals may be useful to introduce new transfusion policies improving the transfusion service. For this type of study, a national electronic database for blood donors and patients is useful for managing the transfusion service and the patients' needs for transfusion. A national transfusion service may also invest in the recruitment of regular blood donors to use electronic crossmatching to improve the safety and reduce the cost of the transfusion service.

In conclusion, the corrective policy of omitting the type and hold step for healthy patients undergoing elective c-sections has greatly improved the utilization of erythrocyte transfusion and reduced the costly consumption of reagents in the blood bank of a maternity hospital. This policy can be adopted in maternity hospital blood banks where which inventory in mainly supplied by replacement blood donors.

\footnotetext{
Acknowledgment. We would like to thank Oxbridge proofreading (https://oxbridgeproofreading.co.uk) for English language editing.
} 


\section{References}

1. Compton ML, Szklarski PC, Booth GS. Duplicate type and screen testing: waste in the clinical laboratory. Arch Pathol Lab Med 2017; 142: 358-363.

2. Mangwana S, Bedi N, Yadav P, Chugh R. Optimization of blood transfusion services: Analysis of blood requisition and utilization practices in cardiac surgical patients in a tertiary care hospital, India. Glob J Transfus Med 2017; 2: 47-51.

3. Khan F, Khan M, Ali A, Chohan U. Estimation of blood loss during caesarean section: an audit. J Pak Med Assoc 2006; 56: 572-575.

4. Singh B, Adhikari N, Ghimire S, Dhital S. Post-operative drop in hemoglobin and need of blood transfusion in cesarean section at Dhulikhel Hospital, Kathmandu University Hospital. Kathmandu Univ Med J 2013; 11: 144-146.

5. Stock O, Beckmann M. Why group \& save? Blood transfusion at low-risk elective caesarean section. Aust NZJ Obstet Gynaecol 2014; 54: 279-282.

6. Collins RA, Wisniewski MK, Waters JH, Triulzi DJ, Yazer MH. Effectiveness of multiple initiatives to reduce blood component wastage. Am J Clin Pathol 2015; 143: 329-335.

7. Yazer MH, Dunbar NM, Cohn C, Dillon J, Eldib H, Jackson $\mathrm{B}$, et al. Blood product transfusion and wastage rates in obstetric hemorrhage. Transfusion 2018; 58: 1408-1413.

8. Woodrum CL, Wisniewski M, Triulzi DJ, Waters JH, Alarcon $\mathrm{LH}$, Yazer MH. The effects of a data driven maximum surgical blood ordering schedule on preoperative blood ordering practices. Hematology 2017; 22: 571-577.

9. Heitmiller ES, Hill RB, Marshall CE, Parsons BJ, Berkow LC, Barrasso CA, et al. Blood wastage reduction using Lean Sigma methodology. Transfusion 2010; 50: 1887-1896.

10. Alghamdi S, Gonzalez B, Howard L, Zeichner S, LaPietra A, Rosen $\mathrm{G}$, et al. Reducing blood utilization by implementation of a type-and-screen transfusion policy. A single-institution experience. Am J Clin Pathol 2014; 141: 892-895.

11. Belayneh T, Messele G, Abdissa Z, Tegene B. Blood requisition and utilization practice in surgical patients at University of Gondar Hospital, Northwest Ethiopia. J Blood Transfus 2013; 2013: 1-5.

12. Malik S, Anwari JS, Alshahrani MM, Alotaibi RA. A retrospective study of transfusion practice: Blood crossmatch ordering in obstetrics. Saudi J Laparosc 2018; 3: 21-24.
13. Chalya PL, Mbunda F, Mabula JB, Massinde AN, Kihunrwa A, Gilyoma JM. Blood transfusion practice in surgery at Bugando Medical Centre in northwestern Tanzania. Tanzan J Health Res 2016; 18: 1-9.

14. Leahy MF, Hofmann A, Towler S, Trentino KM, Burrows SA, Swain SG, et al. Improved outcomes and reduced costs associated with a health-system-wide patient blood management program: a retrospective observational study in four major adult tertiary-care hospitals. Transfusion 2017; 57: 1347-1358.

15. Virk MS, Lancaster D, Quach T, Lim A, Shu E, Belanger G, et al. Optimizing O-negative RBC utilization using a data-driven approach. Transfusion 2020; 60: 739-746.

16. Boisen ML, Collins RA, Yazer MH, Waters JH. Pretransfusion testing and transfusion of uncrossmatched erythrocytes. Anesthesiology 2015; 122: 191-195.

17. Murugesan M, Subbiah SP. Blood requisition and utilization practice in obstetric patients at tertiary care center from South India: A descriptive study. J Appl Hematol [serial online] 2018; 9: 131-135.

18. Smith GA, Gopal-Patel J, Joseph JV, Hobson A, Clarkei K. Audit and review for evidence-based red cell wastage reduction measures. Br J Biomed Sci 2015; 72 : 185-190.

19. Bao Y, Xu C, Qu X, Quan S, Dong Y, Ying H. Risk factors for transfusion in cesarean section deliveries at a tertiary hospital. Transfusion 2016; 56: 2062-2068.

19. Bao Y, Xu C, Qu X, Quan S, Dong Y, Ying H. Risk factor for transfusion in cesarean section deliveries ar a tertiary hosptal. Transfusion 2016; 56: 2062-2068.

20. Kathpalia SK, Chawla J, Harith AK, Gupta P, Anveshi A. Blood transfusion practices among delivery cases: A retrospective study of two years. Med J Armed Forces India 2016; 72: S43-S45.

21. Neb H, Zacharowski K, Meybohm P. Strategies to reduce blood product utilization in obstetric practice. Curr Opin in Anesthesiol 2017; 30: 294-299.

22. Zewdie K, Genetu A, Mekonnen Y, Worku T, Sahlu A, Gulilalt D. Efficiency of blood utilization in elective surgical patients. BMC Health Serv Res 2019; 19: 804.

23. Thompson RM, Thurm CW, Rothstein DH. Interhospital variability in perioperative red blood cell ordering patterns in United States pediatric surgical patients. J Pediatr 2016; 177 : 244-249.e5. 